\title{
Solutions in Constructive Field Theory
}

\author{
Leif Hancox- $\mathrm{Li}^{*}$ \\ bihuili@usc.edu
}

June 7, 2016

\begin{abstract}
Constructive field theory aims to rigorously construct concrete, non-trivial solutions to Lagrangians used in particle physics, where the solutions satisfy some relevant set of axioms. I examine the relationship of solutions in constructive field theory to both axiomatic and Lagrangian quantum field theory (QFT). I argue that Lagrangian QFT provides conditions for what counts as a successful constructive solution, and that it provides other information that guides constructive field theorists to solutions. Solutions matter because they describe the behavior of QFT systems, and thus what QFT says the world is like. Constructive field theory, in incorporating ingredients from both axiomatic and Lagrangian QFT, clarifies existing disputes about which parts of QFT are philosophically relevant and how rigor relates to these disputes.
\end{abstract}

${ }^{*}$ I thank Bob Batterman and Michael Miller for comments and encouragement. Several anonymous referees also contribued valuable comments. 
Copyright Philosophy of Science 2016

Preprint (not copyedited or formatted)

Please use DOI when citing or quoting

\section{Introduction}

To date, philosophers of quantum field theory (QFT) have paid much attention to roughly two kinds of QFT: axiomatic approaches to algebraic QFT (Halvorson and Müger, 2006), and Lagrangian-based QFT as used by particle physicists (Wallace, 2006). Comparatively less attention, however, has been paid to constructive QFT, an approach that aims to rigorously construct non-trivial solutions of QFT for Lagrangians and Hamiltonians that are important in particle physics, ensuring that such solutions satisfy certain axioms. In doing so, constructive QFT mediates between axiomatic approaches to QFT and physicists' Lagrangian-based QFT. It ensures that the various axiom systems have a physically meaningful correspondence with the world. Since we usually take solutions in physical theories to describe the behavior of systems falling under the theory and constructive QFT aims to produce these solutions, constructive QFT deserves some philosophical attention.

Constructive QFT is rather different in approach and aims from what I call, borrowing terminology that stems from Arthur Wightman (1973), axiomatic QFT ${ }^{1}$ Following Wightman and modern practictioners like Summers (2012), I define constructive QFT to be the attempt to rigorously construct interacting models of QFT that correspond to the repertoire of Lagrangians of interest to particle physics. In contrast, axiomatic QFT in this terminology aims to derive results about the structure of QFT independently of any particular Lagrangian. In this paper I examine two aspects of constructive QFT in the functional integral tradition that are relevant to our understanding of the theoretical structure of QFT. The first is the question of what counts as a solution to a specific Lagrangian in constructive QFT. I argue that the criteria for what counts as a solution include some kind of correspondence with perturbation series derived in Lagangian QFT. This correspondence relation is not part of any of the standard axioms of QFT. Thus, the constructed solutions may satisfy "physical criteria" that are not present in axiomatic QFT taken by itself.

The second aspect of constructive QFT in the functional integral tradition that I examine concerns the information that constructive field theorists use to construct solutions. Methods of construction rely heavily on perturbative Lagrangian QFT. The specifics of the regularization methods, counterterms, multiscale expansions, and so on that are used affect the success of the construction. This suggests that a successful construction of physically interesting solutions relies on information that may not be just in the axioms of axiomatic QFT. This information, often derived from Lagrangian QFT, may thus be of philosophical interest.

The paper proceeds as follows. In Section 2 , I clarify what I mean by perturbative Lagrangian QFT, constructive QFT, axiomatic QFT, and other key terms. In Section 3, I provide a quick primer to perturbative Lagrangian QFT, which will help us understand

\footnotetext{
${ }^{1}$ See Section 2 for a fuller explanation of this terminology. As I also explain in that section, my more precise terminology does not always line up with the more casual labels of physicists or the distinctions drawn in the philosophy literature so far.
} 
Copyright Philosophy of Science 2016

Preprint (not copyedited or formatted)

Please use DOI when citing or quoting

some aspects of constructive QFT. In Section 4, I make the two main points described above, then discuss their philosophical implications in Section 5. I conclude with some reflections on general implications for the philosophy of applied mathematics.

\section{Approaches towards Quantum Field Theory: A Primer}

This primer is necessary for two reasons. Firstly, there is a gulf between the methods that physicists take to constitute QFT and the mathematical structures that philosophers of QFT have often taken to constitute QFT. In the philosophy of QFT debate this has become simplified into a contrast between "Lagrangian" QFT and "algebraic" or "axiomatic" QFT (Wallace, 2011; Fraser, 2011, but more nuanced categories are required for my purposes. ${ }^{2}$ Secondly, the dominance of this terminology has led philosophers to gloss over a distinction, long recognized in mathematical physics, between what I will call axiomatic QFT and constructive QFT. This has led to much confusion about the role of perturbative Lagrangian methods in the foundations of QFT. Here, I lay out some terminology that will help clear up such confusions.

Most of the methods described in a standard QFT textbook for physicists involve calculations in perturbative Lagrangian QFT. To remain consistent with previous terminology used by Wallace (2006), I will simply refer to this approach to QFT as Lagrangian QFT, even though physicists also use some methods that are Lagrangian but not perturbative. In Lagrangian QFT, one takes as a baseline an exactly solvable model of QFT in which there are no interactions. Having no interactions, this model is not of direct physical interest. But to solve models in which there are interactions, one can consider the interactions as small perturbations to the exactly solvable non-interacting model. This allows one to apply the apparatus of perturbation theory to obtain what are known as renormalized perturbation series. If we had a justification for the validity of perturbation theory in QFT, then these would be good approximate solutions for the interacting model $\mathrm{H}^{3}$ However, perturbation theory is valid only under certain conditions, and it is hard to verify

${ }^{2}$ To keep things clear, I will use quote marks, as in the previous sentence, to indicate that I am referring to terms used by philosophers so far, without necessarily endorsing those terms. Since I intend to make more fine-grained distinctions than these terms alone allow for, I will mark terms that I endorse using italics.

${ }^{3}$ Throughout this paper, I use the term "model" the way physicists do, as referring to a specific instance of a system falling under a theory. I do not use it in the logicians' sense. The distinction between "solution" and "model" is that "solution" is a kind of success term, whereas it is common for physicists to call something a "model" even if they have not obtained an adequate description of the model which would count as a "solution". For example, Rivasseau (1991, p. 11) uses the term " $\varphi_{4}^{4}$ model" even though there is as yet no such successful construction of such a model, i.e. there is as yet no solution to the model. Gawedzki and Kupiainen (1985, p. 197) speak of models existing "on the level of the formal 
Copyright Philosophy of Science 2016

Preprint (not copyedited or formatted)

Please use DOI when citing or quoting

if these conditions apply in the case of QFT. Furthermore, Lagrangian QFT uses mathematical tools known as Feynman path integrals. While the exact definition of path integrals is still in flux, physicists have devised ways to compute them without adhering to mathematical standards of rigor.

In the interests of clarity, it is worth noting that there are perturbative treatments of QFT that do not coincide with Lagrangian QFT as practiced by particle physicists. For example, there are programs to "rigorously" analyse perturbative Lagrangian QFT (Steinmann, 2000; Salmhofer, 2007), but these form a very small proportion of the work on Lagrangian QFT. The "rigor" in these works concerns manipulating perturbative formalisms according to strict, clearly delineated syntactic rules, which may nonetheless lack non-perturbative justification. This lack means that the formalisms have an indeterminate semantics, since, roughly speaking, we do not know if the perturbative expressions "refer" to anything 4 The use of "rigor" to describe such programs is meant to contrast with the more common practice among physicists of using approximations and cancellations that may not even be syntactically consistent.

Besides these syntactically rigorous perturbative approaches starting from Lagrangians, there are also perturbative approaches within algebraic QFT, which uses a different mathematical framework from Lagrangian QFT (Brunetti, Duetsch, and Fredenhagen. 2009).

Lagrangian QFT has been helpful in providing what physicists regard as approximate solutions to problems in QFT. However, the lack of rigor has driven philosophers to turn their attention primarily to algebraic QFT. "Algebraic" here refers to the mathematics used, namely the use of $C^{*}$ algebras to model local observables. The algebraic approach is based on axioms like the Haag-Kastler axioms. Algebraic QFT is not the only strain of QFT that philosophers regard as sufficiently rigorous. There are other sets of axioms that one can work with, such as the Wightman axioms and the Osterwalder-Schrader (OS)

renormalized perturbation series" although no constructive solution has been found yet for these models. The fact that "solution" is a success term while "model" is not explains language like Wightman's (1973, p. 2) when he writes: "the models under discussion are ones for which it seems very unlikely that an explicit solution will ever be found."

${ }^{4}$ In a simpler mathematical setting, a syntactic rule could be something like Euler's custom of expanding a function out as a "Taylor series" without checking for convergence. Thus, $\frac{1}{1-x}$ could be expanded as $1+x+x^{2}+\ldots$ even outside its conventional radius of convergence (Kline, 1983). This is an instance of using a syntactic rule without semantic justification, because one is mechanically applying rules for manipulating symbols without checking that the symbols refer to anything. To provide a semantic justification, one has to then show that the series actually corresponds to some well-defined quantity. Applying perturbation theory without a non-perturbative justification is similar-one can derive "perturbative expressions" by pure symbolic manipulation, but the resultant expressions may not refer to anything. My use of "syntactic" and "semantic" in this context is consonant with that found in discussions of formalism and other competing philosophies of mathematics, such as Detlefsen (2005, p. 250) and Wilson (2008, pp. 541-3). 
Copyright Philosophy of Science 2016

Preprint (not copyedited or formatted)

Please use DOI when citing or quoting

axioms. The former work in Minkowski space, while the latter work in Euclidean space. They are related to each other by the Osterwalder-Schrader Reconstruction Theorem, as I explain below. The Wightman axioms use a mixture of functional analysis and operator algebras. The OS axioms are the basis of rigorous functional integral approaches to QFT. As with the term "algebraic", this term refers to the mathematics used-in the OS framework, Feynman path integrals used by physicists are reinterpreted in terms of rigorous functional integrals in Euclidean space.

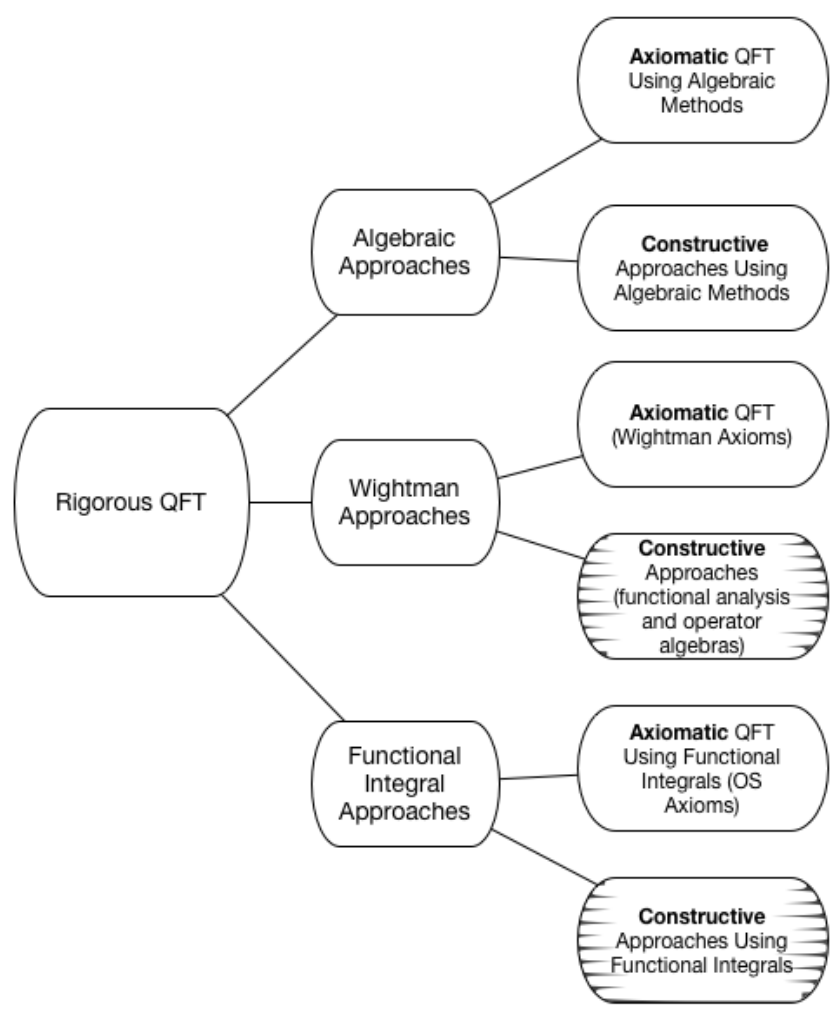

Figure 1: A rough taxonomy of rigorous approaches to QFT. This figure does not aim to be complete, leaving out perturbative algebraic QFT (Brunetti et al., 2009) and "rigorous" perturbative Lagrangian QFT (Steinmann, 2000; Salmhofer, 2007), for example. The embellished bubbles indicate those constructive approaches of central interest to this paper, namely those that seek to rigorously construct models of QFT corresponding to Lagrangians within the usual repertoire of particle physics. As stated in the main text, constructive approaches using algebraic methods are as yet not obviously related to the Lagrangians of particle physics.

Within both the Wightman and functional integral approaches to QFT, we can discern two broad ways of investigating QFT. The first way is what has often been called axiomatic 
Copyright Philosophy of Science 2016

Preprint (not copyedited or formatted)

Please use DOI when citing or quoting

QFT, where the idea is to investigate the consequences of the axioms without relying on the properties of any particular Lagrangians or Hamiltonians. The second way is a constructive approach, which has a very specific aim: to construct concrete, non-trivia 5 examples of models of QFT that satisfy the relevant set of axioms. Figure 1 displays the taxonomy I am describing. For example, one can take a constructive approach in the Wightman framework that aims to construct a $\phi_{2}^{4}$ model and prove that it satisfies the Wightman axioms. This is done by defining a Hamiltonian and showing that it has the requisite algebraic properties (Glimm and Jaffe, 1968). One could also have a corresponding project in the functional integral approach that aims to construct the same $\phi_{2}^{4}$ model using Euclidean functional integrals (Glimm, Jaffe, and Spencer, 1974). Both these projects have the very specific aim of showing that specific models that are used in particle physics are consistent with axiomatic systems, and are to be distinguished from methods that aim to derive general conclusions from axioms without reference to particular Hamiltonians or Lagrangians.

Finally, there is a different kind of constructive project in the algebraic framework, where one constructs models that, in contrast with the Wightman and functional integral constructions, are not guided by Lagrangian QFT (Summers, 2012, p. 38). I will not discuss this kind of constructive work in this paper, as I am focusing on the significance of constructive field theory as a mediator between Lagrangian QFT and axiom systems. While algebraic approaches may one day hope to play such a mediating role, constructive field theory in the functional integral tradition is at present much more in contact with Lagrangian QFT ${ }^{6}$ The lack of this mediating role for such purely algebraic constructions makes their philosophical significance potentially very different from that of Wightman and functional integral constructions.

I call the project of constructing non-trivial solutions to models of QFT, where such models are derived from the usual repertoire of Lagrangian QFT as practiced by physicists, constructive QFT. In doing so I am merely using terminology that dates from Wightman and is still used by contemporary mathematical physicists. In an introductory article on constructive field theory, Wightman writes:

Constructive quantum field theory differs from axiomatic field theory in that it attempts to construct the solutions of specific concrete model theories, typically simplified analogues of self-coupled meson theories or Yukawa theories of meson-baryon couplings. On the other hand, axiomatic field theory customarily attempts to make statements about all theories satisfying certain quite general assumptions (Wightman, 1973, p. 1).

Wightman reiterates this distinction in other articles (Wightman, 1969, 1976), emphasizing

\footnotetext{
${ }^{5}$ In QFT, both physicists and mathematicians use "non-trivial" to refer to models containing interactions, ruling out those that contain only free fields.

${ }^{6}$ Thus Jaffe and Witten's description of the Clay Institute's Millenium Problem of constructing a four-dimensional interacting Yang-Mills theory with a mass gap, for example, focuses on attempts at the problem from the functional integral point of view (Jaffe and Witten, 2005).
} 
Copyright Philosophy of Science 2016

Preprint (not copyedited or formatted)

Please use DOI when citing or quoting

each time that the concrete models chosen are those from the repertoire of Lagrangian QFT.

A more modern review of QFT confirms that Wightman's distinction still exists. Fredenhagen, Rehren, and Seiler (2006, p. 4) write that "The power of the axiomatic approach resides not least in the ability to derive structural relations among elements of the theory without the need to actually compute them in a model." They then continue in the section on constructive QFT:

The axiomatic approach, on the other hand, does not answer the question whether the axioms are not empty, i.e. whether any nontrivial QFTs satisfy them. The constructive approach is in principle addressing both of these problems. On the one hand it attempts to show that the axiomatic framework of QFT is not empty, by mathematically constructing concrete nontrivial examples satisfying these axioms, and on the other hand it provides non-perturbative approximation schemes that are intimately related to the attempted mathematical constructions; the prime example[sic] are the lattice approximations to QFTs (Fredenhagen et al., 2006, p. 14).

Constructive QFT is thus very different from axiomatic QFT in its aims and methodology. Philosophers have for the most part focused only on axiomatic QFT or perturbative Lagrangian QFT/7 Looking at the rigorous approaches, when philosophers do consider models of QFT at all, these have been non-interacting models, which are not the focus of constructive QFT $]^{8}$ Constructive QFT contains the best attempts so far to construct rigorous solutions to Lagrangian models, having done this successfully for several systems with dimensions other than four 9 Since the actual world is four-dimensional, the main aim is to extend these successes to the case of four dimensions, but this has not been achieved yet for Lagrangians resembling those used by particle physicists.

One can think of constructive QFT as performing the important task of ensuring that that our axiom systems have a physically meaningful correspondence with the world. That is, since the confirmation of QFT proceeds via deriving the consequences of specific

${ }^{7}$ For examples of the former, see Halvorson and Clifton (2002) and Baker and Halvorson (2010). For examples of the latter, see Wallace (2006) and Teller (1997). There are one or two exceptions that perhaps fall in a middle ground, such as Fraser (2008).

${ }^{8}$ See Ruetsche (2011) and Clifton and Halvorson (2001) for philosophical work on non-interacting models. To some extent, whether or not one includes non-interacting models in the category of "constructive QFT" is a terminological issue. I am merely following one (sensible) convention, adopted by Fredenhagen et al. (2006) in the quote above, who specify that the models have to be non-trivial. Insofar as the main purpose of constructive QFT is to mediate between empirical data and axioms, it makes sense to consider its main focus to be on interacting models.

${ }^{9}$ The construction can also be done with Hamiltonians, but in either case, constructive QFT is about constructing models of QFT containing specific interactions, whereas the whole point of axiomatic QFT is to avoid reference to specific interactions. To avoid textual unwieldiness, I will just say "Lagrangian models" from now on with the understanding that Hamiltonian models are also allowed. 
Copyright Philosophy of Science 2016

Preprint (not copyedited or formatted)

Please use DOI when citing or quoting

Lagrangian models, we need to show that our axiomatic systems can accommodate such models. That is why constructive QFT does not just attempt to construct models of random Lagrangians, but focuses on specific ones, such as the ones in four dimensions, the ones of Yang-Mills theory, the Higgs interaction, and so on ${ }^{10}$ In philosophy of language terms, we can think of constructive QFT as attempting to show that our axiomatic systems are not just games of symbols played according to fixed syntactic rules; that they have external validity in addition to the internal consistency that their syntax provides. External validity lies in the connection to empirical success, which is why Wightman emphasizes the connection to heuristic Lagrangian QFT ${ }^{11}$

A few caveats ought to be made at this point. Firstly, my categorization of different approaches to QFT does not rule out borderline cases. There are constructive projects combining functional integral methods with Wightman-oriented methods, and others combining algebraic and Wightman methods (Summers, 2012, pp. 4, 8, 12). Secondly, there is some variation in terminology within the mathematical physics community. While I follow Wightman and others in classifying under "axiomatic QFT" those approaches that do not rely on the properties of specific Lagrangians or Hamiltonians, Arthur Jaffe (1969) has written an article titled "Whither Axiomatic Field Theory?" in which he discusses what I here call constructive field theory. Similarly, Huzihiro Araki (1972) discusses constructive QFT as an "area" of axiomatic field theory. However, both Jaffe and Araki are in agreement that constructive field theory involves constructing models of QFT, these models being concrete realizations of particular Lagrangians or Hamiltonians. Jaffe (1969, p. 576) writes: "Constructive field theorists have approached this problem with Lagrangian field theories and attempted to solve particular model Lagrangians." Araki (1972, p. 1) writes: "[Constructive field theory] is an attempt to construct a quantum field theory for a given interaction such as $\phi^{4}$ and $\bar{\psi} \psi \phi$ in a mathematically satisfactory manner." Thus, there is a consensus in the literature that constructive field theory, whether one classifies it under the axiomatic program or not, is an approach which is defined by its attempt to construct models of particular interactions, represented by Lagrangians or Hamiltonians.

In short, we can clearly recognize, and the community does recognize, a distinction between approaches that proceed from the axioms alone and approaches that work with concrete models, where these models are taken from Lagrangian QFT. I have, following one convention, chosen to call the former axiomatic and the latter constructive.

Terminological disputes aside, it still remains a fact that philosophers have paid little attention to the latter, and that the latter affords many interesting connections with

\footnotetext{
${ }^{10}$ Later, we will see that one of the main problems in constructive QFT is figuring out in what sense the "Lagrangians" used in constructive QFT correspond to the semi-formal "Lagrangians" used in Lagrangian QFT.

${ }^{11}$ In an applied math context like QFT, external validity lies in ensuring that our language latches on to the world in some sense. I leave it open what this "latching on to" relation is in the details. The simplest account of it would be some kind of isomorphism to mathematical objects (Pincock, 2004), but more complex views are possible (Batterman, 2010).
} 
Copyright Philosophy of Science 2016

Preprint (not copyedited or formatted)

Please use DOI when citing or quoting

Lagrangian QFT that are easy to ignore if one concentrates on the former as the paradigm example of rigorous QFT.

Now that we have a clear terminology, I will move on to provide more details about the general approach of Lagrangian QFT. This is necessary to explain later how solutions in constructive QFT relate to Lagrangian QFT.

\section{Perturbative Solutions in Lagrangian Quantum Field Theory}

For physicists, the dynamics of QFT are derived from a quantity known as the action:

$$
S[\phi]=\int d^{4} x \mathcal{L}\left(\phi(x), \partial \phi(x) / \partial x^{\mu}\right),
$$

where $\mathcal{L}$ is the Lagrangian of the quantum field $\phi$. The square brackets indicate that $S[\phi]$ is a functional, not a function. It depends on the values of $\phi$ everywhere, not just at some particular point of spacetime. The form of the Lagrangian is based on considerations of the kind of interactions we expect in the system of interest, and on the symmetries we expect the system to obey. The action is closely related to the classical field equations by way of a variational principle. Essentially, postulating that $S[\phi]$ has to be at an extremum will lead us to the classical field equations. Thus, the action can be regarded as representing the dynamics of the theory.

From the action we get the partition function, which is defined as follows:

$$
Z=\int \mathcal{D} \phi e^{S[\phi]}
$$

Here, the integral is a functional integral, meaning that we are integrating over all possible combinations of $\phi$ 's values over spacetime ${ }^{12}$ Once we know $Z$, we can typically calculate all the empirical quantities associated with that particular Lagrangian, such as the scattering cross-sections found in particle physics experiments. Thus, finding an expression for $Z$ is a primary goal of much of QFT. A successful evaluation of $Z$ is considered to be a solution of Lagrangian QFT.

\subsection{Perturbative Renormalization}

Equation 1 can be given a straightforward finite, analytic expression when the action involved is that of a free scalar field with no interactions, also known as a "Gaussian" field.

\footnotetext{
${ }^{12}$ Later, we will see that the difficulties of defining a measure for this integral is one reason why Lagrangian QFT is commonly thought to be unrigorous. Constructive field theory is distinguished from Lagrangian QFT in part because it attempts to define a measure rigorously.
} 
In this case, $\mathcal{L}=\frac{1}{2}\left((\partial \phi)^{2}-m^{2} \phi^{2}\right)$. For interacting fields, physicists typically use perturbation theory to evaluate the path integrals. Since the path integral for the free field has a known analytic expression, the perturbations are applied using the free field case as a reference-we consider the interaction as a small perturbation to the free field Lagrangian. The following example illustrates how this is done in a simple case.

Suppose a small ${ }^{13}$ interaction $-\frac{\lambda}{4 !} \phi^{4}$ is added to the free field Lagrangian, so that $\mathcal{L}=\frac{1}{2}\left((\partial \phi)^{2}-m^{2} \phi^{2}\right)-\frac{\lambda}{4 !} \phi^{4}$. This is the Lagrangian of the so-called $\phi^{4}$ theory, which describes a self-interacting scalar field. The partition function is

$$
Z=\int \mathcal{D} \phi e^{\int d^{4} x\left(\left((\partial \phi)^{2}-m^{2} \phi^{2}\right)-\frac{\lambda}{4 !} \phi^{4}\right)} .
$$

Assuming $\lambda$ to be small, we then convert the $e^{-\frac{\lambda}{4 !} \phi^{4}}$ factor into a Taylor series in $\lambda$ :

$$
Z=\int \mathcal{D} \phi\left(1-\frac{\lambda}{4 !} \int_{x_{1}} \phi^{2}\left(x_{1}\right) d x_{1}+\frac{1}{2}\left(\frac{\lambda}{4 !}\right)^{2} \int_{x_{1}, x_{2}} \phi^{4}\left(x_{1}\right) \phi^{4}\left(x_{2}\right) d x_{1} d x_{2}+\cdots\right) e^{\int d^{4} x\left((\partial \phi)^{2}-m^{2} \phi^{2}\right)},
$$

where I have included only the first two terms of the Taylor series to illustrate the general rule.

Unlike in the free field case, when evaluating path integrals such as the above, several mathematical problems arise. One is how to define the measure $\mathcal{D} \phi$. Physicists calculate $Z$ without a precise definition of the measure. Constructive field theorists attempt to define it rigorously. The second class of problems surrounds infinities known as divergences that arise in one's calculations. These divergences occur in two forms. Firstly, individual terms in the perturbation series might diverge. Secondly, the perturbation series itself may not be a convergent series, though it may be an asymptotic series that can be summed by special summation methods.

To deal with the first type of divergence, physicists perform the following procedures:

1. Regularization, which is the reduction of the number of degrees of freedom in the problem by adding momentum cutoffs, dimensional regularization, or moving to a lattice formulation;

2. Addition of counterterms to compensate for the regularization.

A theory is said to be perturbatively renormalizable if after regularization and the adding of counterterms, the number of coupling parameters in the theory is finite and constant at each order in perturbation theory. Another way of putting this is that renormalization occurs when the effects of regularization and the adding of

\footnotetext{
${ }^{13}$ In the perturbative context, a small interaction is one for which the coupling constant, when expressed in dimensionless units, is less than 1 .
} 
Copyright Philosophy of Science 2016

Preprint (not copyedited or formatted)

Please use DOI when citing or quoting

counterterms are absorbed entirely by changing only the values of a finite number of coupling parameters. For example, in the $\phi^{4}$ model, the Lagrangian we start with before regularization and renormalization is $\mathcal{L}=\frac{1}{2}(\partial \phi)^{2}-\frac{1}{2} m^{2} \phi^{2}-\frac{\lambda}{4 !} \phi^{4}$. In carrying out dimensional regularization, we first go from 4 dimensions to $4-\varepsilon$ dimensions. This introduces a new scale $\mu$ and an $\varepsilon$ exponent into the Lagrangian, so that $\mathcal{L}=\frac{1}{2}(\partial \phi)^{2}-\frac{1}{2} m^{2} \phi^{2}-\mu^{2 \varepsilon} \frac{\lambda}{4 !} \phi^{4}$ after regularization. After both dimensional regularization and renormalization, we obtain a renormalized Lagrangian $\mathcal{L}_{r e n}$ with counterterms $\mathcal{L}_{c t}($ Ramond, 1981, p. 132):

$$
\mathcal{L}_{r e n}=\mathcal{L}+\mathcal{L}_{c t},
$$

where

$$
\begin{gathered}
\mathcal{L}_{\text {ren }}=\frac{1}{2}\left(\partial \phi_{0}\right)^{2}-\frac{1}{2} m_{0}^{2} \phi_{0}^{2}-\frac{\lambda}{4 !} \phi_{0}^{4}, \\
\mathcal{L}=\frac{1}{2}(\partial \phi)^{2}-\frac{1}{2} m^{2} \phi^{2}-\mu^{2 \varepsilon} \frac{\lambda}{4 !} \phi^{4},
\end{gathered}
$$

and

$$
\mathcal{L}_{c t}=\frac{1}{2} A(\partial \phi)^{2}-\frac{1}{2} m^{2} B \phi^{2}-\mu^{2 \varepsilon} \frac{\lambda}{4 !} \phi^{4} .
$$

The coupling parameters $\phi, m$ and $\lambda$ have been renormalized as follows:

$$
\begin{gathered}
\phi_{0}=(1+A)^{1 / 2} \phi, \\
m_{0}^{2}=m^{2} \frac{1+B}{1+A}, \\
\lambda_{0}=\lambda \mu^{2 \varepsilon} \frac{1+C}{(1+A)^{2}} .
\end{gathered}
$$

$A, B$ and $C$ are constants that determine how the coupling parameters are renormalized. As one can see, the counterterms that are added preserve the form of the interactions in the original Lagrangian, so that one has to change only the existing finitely many coupling parameters in $\mathcal{L}$ in order to obtain $\mathcal{L}_{r e n}$. This is why the $\phi^{4}$ theory is said to be perturbatively renormalizable.

The perturbation series with the new coupling parameters is known as the renormalized perturbative series, which has the same terms as the original one, but with the renormalized coupling parameters instead of "bare" coupling parameters. This series is the basis of many, if not most, successful empirical predictions in QFT. However, because it is unclear whether the series converges or whether it is asymptotic to some function we can derive in constructive field theory, the series is 
Copyright Philosophy of Science 2016

Preprint (not copyedited or formatted)

Please use DOI when citing or quoting

purely "formal", in the sense of being a mere string of symbols that may have no mathematical referent 14

We will see next that the renormalized perturbation series also plays an important role in constructive QFT, forming part of the success conditions for a constructive solution.

\section{Constructive Solutions in Quantum Field Theory}

Constructive QFT is the effort to construct, according to the usual standards of mathematical rigor, solutions to Lagrangian models in QFT, and to prove that these solutions satisfy certain axioms that we expect to apply to all Lagrangian models. In the functional integral tradition, the usual method is to shift from Minkowski spacetime to Euclidean spacetime. This is done by a Wick rotation, where one replaces the time parameter in Minkowski spacetime with an imaginary time variable $-i t$. This turns out to be extremely helpful for constructing the functional integral measures needed for Equation $1-$ many constructions that are possible in Euclidean spacetime have not been accomplished directly in Minkowski spacetime.

In Euclidean field theory, the relevant axioms to be satisfied are the OS axioms. These axioms state the properties that the Schwinger functions of the model must satisfy. The Schwinger functions are as follows:

$$
S_{N}\left(z_{1}, \ldots, z_{N}\right)=Z^{-1} \int \prod_{j=1}^{N} \phi\left(z_{j}\right) e^{-\int V(\phi(x)) d x} d \mu_{0}(\phi),
$$

where

$$
Z=\int e^{-\int V(\phi(x)) d x} d \mu_{0}(\phi) .
$$

$V$ is the interaction part of the Lagrangian. The measure $d \mu_{0}(\phi)$ is a Gaussian measure on the Schwartz space of rapidly decreasing functions. This measure accounts for the free field part of the Lagrangian, so that Equation 4 is a translation of Equation 1. even though the former does not contain the free-field portion in the exponential (Sénéor, 1988, p. 23).

The OS axioms are related to the Wightman axioms by the Osterwalder-Schrader Reconstruction Theorem, which states that any set of

\footnotetext{
${ }^{14}$ It is not an entirely unmotivated string of symbols, since it is derived by an extension of the syntax of perturbation theory to a case where perturbation theory might apply. However, without a proof that perturbation theory is valid in this context, we do not know if this syntactic expression has a mathematical referent.
} 
Copyright Philosophy of Science 2016

Preprint (not copyedited or formatted)

Please use DOI when citing or quoting

functions satisfying the OS axioms determine a unique Wightman model whose Schwinger functions form that set (Rivasseau, 1991, p. 22). This theorem allows constructive field theorists in the functional integral tradition to work in Euclidean spacetime, knowing that any successful construction satisfying the OS axioms can be translated into a successful construction satisfying the Wightman axioms. This way, they can exploit the advantages of Euclidean spacetime when it comes to defining a measure for Equation 1, while ensuring that they are still effectively constructing models that can live in Minkowski spacetime.

A solution in constructive QFT would be a construction of Schwinger functions that satisfy the OS axioms and some other conditions (to be described below). Before evaluating Equation 3, however, we have to provide a meaning to it. We have to define what the integral over function space could possibly mean. This is not a straightforward task because for interacting fields, we encounter the same problems as in Lagrangian QFT with regularization and counterterms ${ }^{15}$ These questions of defining the functional integral will be discussed in Section 4.2 . Additionally, to make sure that the construction is indeed a solution that corresponds to an interaction of interest in Lagrangian QFT, we have to show that the construction has some kind of correspondence to the renormalized perturbation series that physicists derive for the corresponding Lagrangian in Lagrangian QFT ${ }^{16}$ Crucially, this latter property, which I will discuss next in Section 4.1, is not part of the OS axioms.

\subsection{Correspondence with Physicists' Perturbative Methods}

A large part of the motivation of constructive QFT is to show that the Lagrangian models that physicists have used with such empirical success do in fact have solutions that are rigorously defined. Physicists have long used their own "solutions" in the form of renormalized perturbation series, but, as explained above, these are obtained in mathematically dubious ways. Nonetheless, the empirical success of these series suggests that they are like solutions. We want the rigorously constructed solutions of constructive QFT to be connected, somehow,

\footnotetext{
${ }^{15}$ There are also non-perturbative issues in defining the functional integral, which I will not discuss in this paper, such as trying to put bounds on regions in which $\phi$ is large (otherwise known as the large field problem). These are usually dealt with by multiscale expansions (Rivasseau, 1999, pp. 6-7).

${ }^{16}$ This correspondence is a kind of "ultimate" success condition. Much of constructive field theory consists of constructing toy models that resemble Lagrangians used in particle physics but are not necessarily identical to them, such as "wrong sign" planar four-dimensional $\phi^{4}$ (Rivasseau, 1991, Chapter II.5). These toy models serve as a useful test ground for techniques that may help us to construct solutions that do correspond to Lagrangians used in four-dimensional particle physics.
} 
Copyright Philosophy of Science 2016

Preprint (not copyedited or formatted)

Please use DOI when citing or quoting

with this empirical success, and thus with the renormalized perturbation series. We also want to make sure that the Lagrangian we construct a solution for is "the same" Lagrangian that the physicists use. This is more involved than it appears because the physicists' Lagrangian is not mathematically well-defined as it stands. While physicists can write down the formal identity $\mathcal{L}=\frac{1}{2}\left((\partial \phi)^{2}-m^{2} \phi^{2}\right)-\frac{\lambda}{4 !} \phi^{4}$, say, this is not enough to constitute defining $\mathcal{L}$ rigorously, because the rules with which they manipulate $\mathcal{L}$ are not well-defined.

Suppose, then, that I show that for a particular $\mathcal{L}$, the right-hand side of Equation 3 can be rigorously constructed. Wightman asks:

How can you answer the question, "What problem did you solve?" The answer would be "I solved the problem of showing that certain limits existed and that they had certain properties." But you never write down any condition which fixed the theory you were talking about. We argue, of course, that conventional renormalized theories are fixed by choosing coupling constants and masses (Wightman, 1986, p. 226) 17

The choice of coupling constants and masses is fixed by the renormalized perturbation series of the Lagrangian. In other words, to make sure that we have solved a Lagrangian that "is" the $\phi^{4}$ Lagrangian, say, we have to make sure that our construction somehow reproduces the same coupling constants and masses that the renormalized perturbation series for the $\phi^{4}$ interaction has.

Wightman makes a similar point in an earlier paper, when he asks what it means to "solve" a theory:

Of course, one can answer by saying one wants to construct a field satisfying the usual axioms of quantum field theory that somehow solves the equations of the model, but in fact, one wants much more than that (Wightman, 1973, p. 3).

The first of the "much more than that" objectives is as follows:

1. Renormalized Hamiltonian: $H_{\text {ren }}$ in the space of physical states. How one recognizes that it is the renormalized Hamiltonian for the somewhat illdefined[sic] model one starts out from is a question about which much will be said later (Wightman, 1973, p. 3, underlining in original, emphasis mine)

On a similar note, James Glimm asks:

$[\mathrm{H}]$ ow does one recognize a solution to the problem? In general terms a solution should possess as far as possible the expected properties. For

\footnotetext{
${ }^{17}$ Wightman is using "theory" where I would use "model".
} 
Copyright Philosophy of Science 2016

Preprint (not copyedited or formatted)

Please use DOI when citing or quoting

example the Taylor coefficients [of the perturbation series] should agree with the standard formulae (Glimm, 1969, p. 103).

For a more modern source, we can look to Gallavotti and Rivasseau (1984, p. 209), who point out that a $\phi_{4}^{4}$ theory cannot be just something that satisfies certain axioms, is four-dimensional, and is scalar, but must also "correspond at least in some sense to a $\phi_{4}^{4}$ lagrangian[sic].' 18 They then add that given the historical success of perturbation theory in other field theories, this notion of correspondence should include at least some notion of being asymptotic to the renormalized perturbation series.

Much work in constructive QFT revolves around defining this correspondence relation. One option is to say that the correspondence exists if the rigorously constructed $Z$ is asymptotic to the renormalized perturbation series. A function is asymptotic to a series expansion if, roughly speaking, the successive terms of the series provide an increasingly accurate description of how quickly the function grows. Asymptotic series need not be convergent. ${ }^{19}$ However, as we come up with new ways to sum series, we also find find new ways to prove asymptoticity. Constructive QFT is engaged in finding new ways to sum physicists' perturbation series so as to relate them to the non-perturbative constructions of $Z$. This means that the notion of "correspondence" is expanding as we find new ways to sum perturbative series and possibly prove asymptoticity. There is also the possibility that the notion of correspondence that would work is one that is weaker than convergence (of a series to a function) but stronger than asymptoticity. Barry Simon (1982, p. 6) for example proposes the notion of a strong asymptotic series for this purpose. This latter kind of series has the advantage that at most one function $f$ has a given series as a strong asymptotic series, whereas multiple functions may have the same series as an asymptotic series (Strocchi, 2013, p. 1).

In constructive field theory, the most commonly used variety of strong asymptotic summability is that of Borel summability. This has been used in many important constructions, such as that of Magnen and Sénéor (1977). Consider an asymptotic series $\sum_{k}^{\infty} f_{k} g^{k}$, associated with a function $f(g)$, of zero radius of convergence. If the divergence is caused by the coefficients $f_{k}$ growing factorially, then we can obtain a series with non-zero radius of convergence by dividing each term in the expansion by $k$ !. This gives us the Borel transform of the function, $B(g)=\sum_{k}^{\infty} B_{k} g^{k}$, where $B_{k}=f_{k} / k ! . f(g)$ is Borel summable if the Borel transform can be summed and analytically continued over the entire positive real axis. We

${ }^{18}$ Following standard practice in QFT, adding a subscript $n$ to an interaction, as in $\phi_{n}^{4}$, indicates that we're working in $n$-dimensional spacetime.

${ }^{19}$ See Erdelyi 2010) for a technical definition of an asymptotic expansion. 
Copyright Philosophy of Science 2016

Preprint (not copyedited or formatted)

Please use DOI when citing or quoting

can then recover the original function $f(g)$ via the integral $f(g)=\int_{0}^{\infty} d t e^{-t} B(g t)$. The $f(g)$ thus recovered is the Borel sum of the Borel summable series $\sum_{k}^{\infty} f_{k} g^{k}$. In this way, we can associate an analytic function $f$ with the asymptotic series $\sum_{k}^{\infty} f_{k} g^{k}$, just as a convergent Taylor series can be associated with an analytic function. Part of the task of constructive field theory is to try to do this for renormalized perturbation series.

There are ways to modify or generalize this notion of Borel summability. For example, there is the Borel-Leroy transform (Kleinert and Schulte-Frohlinde, 2001, p. 293) and distributional Borel summability (Caliceti, Grecchi, and Maioli. 1986). It has been suggested that modified notions of Borel summability may be required to deal with certain divergences, known as "renormalons", of the $\phi_{4}^{4}$ model (Caliceti et al., 1986, p. 163). The crucial goal is to find a way of associating a unique function with the renormalized perturbation series, and some version of Borel summability is the main candidate so far.

\subsection{Assigning a Meaning to the Functional Integral}

Having described how the generating functional $Z$, which appears in the Schwinger functions, needs to correspond to physicists' renormalized perturbation series, I will now highlight more ways in which a successful construction requires information that is not in the OS axioms.

As I have emphasized, Equation 3 by itself is a mere formalism because we have not yet assigned a meaning to the functional integral. Because it is a mere formalism, there are multiple ways through which we may try to define it, though not all of them might work.

One major barrier to defining the functional integral is that many of the more naive attempts to define it imply that the integral is divergent and therefore apparently not meaningful. Because of this, one has to regularize the integral with both infrared (low-momentum) and ultraviolet (high-momentum) cutoffs, then make sure that the integral has a well-defined limit when the cutoffs are removed. If the limit exists, then the functional integral is meaningful. In constructive QFT the regularization methods used include lattice regularization and Pauli-Villars regularization (Gallavotti and Rivasseau, 1984, p. 187). While different regularizations might seem like just different means to get the same product, i.e. the Schwinger functions, this is not the case. Sometimes one method of construction can work where others fail. The debate about whether the $\phi_{4}^{4}$ model is trivial is instructive about this feature of constructive QFT. There are some heuristic arguments from Lagrangian QFT that $\phi_{4}^{4}$ is trivial (Callaway, 1988). Constructive field theorists have attempted more rigorous proofs of triviality, but the multitude 
Copyright Philosophy of Science 2016

Preprint (not copyedited or formatted)

Please use DOI when citing or quoting

of possible methods of constructing $\phi_{4}^{4}$ is proving to be an obstacle. Gallavotti and Rivasseau (1984, p. 210) argue that to prove that $\phi_{4}^{4}$ theory is trivial, one has to prove that all possible ways of taking the continuum limit, regularizations, bare Lagrangians, and so on do not work, but to prove that a solution exists, one needs to show only that a particular way of construction works. Similarly, Wightman (1986, p. 221) downplays the significance of failed constructions on the basis that other approximation methods could converge to a non-trivial solution. Douglas (2011. Slide 18) points out that the proof by Aizenman (1981) of the triviality of $\phi^{4}$ in more than four dimensions is not a complete proof of triviality, because it's possible that alternative methods of construction may produce non-trivial solutions. This is corroborated by Gallavotti (1985, p. 26), who thinks that there are clues that Aizenman's lattice regularization is inadequate.

Roland Sénéor (1988) describes another example where the choice of regularization affects the success of the construction. He considers the problem of constructing a finite volume Yang-Mills Euclidean theory. He argues that a method of regularization which preserves gauge invariance fails, while one which initially breaks gauge invariance succeeds - the gauge invariance being recovered when the continuum limit is taken.

Besides regularization methods, there are other dimensions on which constructions of $Z$ can differ. One such dimension is the counterterms that we add to the "bare Lagrangian". In QFT, the Lagrangian is a scale-dependent entity. That is, the form of the Lagrangian changes depending on the momentum scale at which the phenomenon of interest occurs. In evaluating the right-hand side of Equation 3 , one initially takes $\mathcal{L}$ to be some "bare Lagrangian" of a certain form, which will however have to be modified in the process of the construction, due to the presence of multiple scales in the functional integral. In four dimensions, this modification takes the form of "counterterms" which one adds to the bare Lagrangian. The nature of these counterterms is generally determined by studying the calculational methods that physicists have found to work in Lagrangian QFT (Rosen, 1973, p. 87, Summers, 2012, pp. 8, 17, 21, 22). Neither the axioms nor Equation 3 tell us which counterterms to use. This is yet another dimension on which Equation 3 is really just a bare formalism to be filled in with further information, rather than something mathematically unambiguous 20

\footnotetext{
${ }^{20}$ It is important to note that even though the choice of counterterms is determined using information from non-rigorous perturbation theory, the construction itself is still rigorous. In perturbation theory the unrigorous part is not the addition of counterterms per se but rather the taking of limits on perturbation series, the assumption that perturbation series have a meaning without checking conditions of validity, the failure to define functional integrals properly, and various other things. Constructive field theory patches these gaps, but does not eschew the use of counterterms.
} 
Copyright Philosophy of Science 2016

Preprint (not copyedited or formatted)

Please use DOI when citing or quoting

To complicate matters further, what we really want from a solution is for the continuum Lagrangian, that is, the one we get after taking the relevant limits on the cutoff integral or the lattice, to correspond to the Lagrangian that is used by physicists in Lagrangian QFT. This can happen even if the bare Lagrangian one starts with in constructive QFT contains terms different from those in the bare Lagrangian that physicists use. The attempts to prove the trivality of $\phi_{4}^{4}$ provide possible examples of this. Gallavotti and Rivasseau (1984, p. 186) find that cut-off $\phi_{4}^{4}$ models which have a negative coupling constant could converge, after removal of the cut-off, to the usual renormalized perturbation series with positive coupling constant. They further argue that even if one fails to construct the $\phi_{4}^{4}$ model, say, using an initial Lagrangian $\mathcal{L}=\frac{1}{2}\left((\partial \phi)^{2}-m^{2} \phi^{2}\right)-\frac{\lambda}{4 !} \phi^{4}$, which is the expression that physicists use in perturbation theory, this does not rule out the possibility of constructing the same model using an initial Lagrangian $\mathcal{L}=\frac{1}{2}\left((\partial \phi)^{2}-m^{2} \phi^{2}\right)-\frac{\lambda}{4 !} \phi^{4}-\mu \phi^{6}$ in a lattice context Gallavotti and Rivasseau 1984, pp. 211-212) ${ }^{21}$ Both these lattice Lagrangians could give rise to Zs that are asymptotic to the renormalized perturbation series that physicists calculate from the bare Lagrangian $\mathcal{L}$ using perturbation theory. Thus, they could both be possible starting points for a construction of $\phi_{4}^{4}$. This creates further ambiguities in the construction process, since it is not even clear what form of Lagrangian should be the input for the right-hand side of (3). There is a kind of underdetermination problem here: the formalism of Equation (3) and the criterion of correspondence to renormalized perturbation series are insufficient to determine the raw materials with which the solution is to be constructed.

In short, the success of a construction in constructive QFT depends on obtaining for $Z$ some kind of correspondence with the renormalized perturbation series, finding a suitable regularization, incorporating counterterms suggested by perturbation theory, and possibly even figuring out what Lagrangian to put into Equation 3. All these factors influence the nature of the solution even though they are external to the OS axioms. Indeed, the contribution of perturbation theory to defining what it means to be a solution is particularly interesting, since part of the prior philosophical debate on QFT concerned whether "Lagrangian QFT" is relevant to the philosophy of QFT (Fraser, 2011). If we accept the solutions of constructive QFT as relevant to philosophers, then the importance of perturbative methods to these solutions suggests that "Lagrangian QFT" is indeed relevant.

${ }^{21}$ Note that this is separate from the counterterms issue. Counterterms are the difference between the "bare" Lagrangian and the renormalized Lagrangian, whereas this is a question of what happens when we move from the lattice to the continuum, not what happens with renormalization. 
Copyright Philosophy of Science 2016

Preprint (not copyedited or formatted)

Please use DOI when citing or quoting

\section{Philosophical Implications}

The features of constructive QFT I have discussed are of philosophical interest for the following reasons. Firstly, while some philosophers attach little interpretive significance to perturbative solutions in Lagrangian QFT due to their lack of rigor, constructive QFT attempts to remedy this by providing rigorous non-perturbative analogues of these perturbative solutions ${ }^{22}$ Generally speaking, these solutions describe the behavior of QFT systems and thus are of interpretive interest to philosophers of QFT.

Secondly, the solutions of constructive QFT are a way to provide the axioms of QFT with physical content. Perturbative Lagrangian QFT is confirmed by its empirical success, but axiomatic QFT cannot be confirmed in as direct a manner. Constructive QFT attempts to ensure that axiomatic QFT can be linked to Lagrangian QFT and thus indirectly confirmed. It does this by trying to show that we can construct solutions that satisfy the relevant axioms, and that these solutions are in some sense "the same" solutions as the perturbative solutions of Lagrangian QFT. Constructive QFT is thus important to those who are interested in confirming or improving extant axiom systems.

Thirdly, critics of Lagrangian QFT like Fraser (2011) have dismissed the relevance of Lagrangian QFT to the "theoretical content" of QFT on the basis of Lagrangian QFT's lack of rigor. Furthermore, Fraser marshalls the successes of constructive QFT to defend rigorous approaches to QFT against Lagrangian QFT. Against this, I have argued that information from Lagrangian QFT is key to the success of constructive QFT. With the example of constructive QFT in the functional integral tradition, we can see that unrigorous Lagrangian QFT is extremely relevant to the rigorous program of constructive QFT, providing essential guidance to a correct construction. By Fraser's own lights, constructive QFT might illuminate the theoretical content of QFT. But given how constructive QFT relies on information from Lagrangian QFT, perhaps Lagrangian QFT itself might contribute to theoretical content.

Fraser acknowledges that constructive QFT attempts to implement analogues of renormalization group (RG) methods that are used in Lagrangian QFT. However,

\footnotetext{
${ }^{22}$ I use the term "analogues" here, rather than saying that perturbative solutions are approximations to constructive solutions, for the following reason. As emphasized in Sections 3.1 and 4.1 , from a mathematical point of view, perturbation series are formal expressions that, in the absence of the right kind of correspondence to a constructive solution, do not have a mathematical referent. It seems inappropriate to call an expression with no meaning an approximation. Of course, should the constructive project succeed, then we will have some way to assign meaning to perturbative expressions, and it may then be appropriate to call them approximations.
} 
Copyright Philosophy of Science 2016

Preprint (not copyedited or formatted)

Please use DOI when citing or quoting

she interprets this fact as showing that RG methods "concern the empirical structure of the theory rather than the theoretical content" (Fraser, 2011, p. 132). In my view, it's unclear that the elements of Lagrangian QFT borrowed by constructive QFT fall on the "empirical" rather than "theoretical" side of the divide-even supposing there is such a dichotomy. The implementation of the RG concerns scaling properties of fields, which is not something that is merely empirical, since it concerns more than just scattering matrices. Without further argument, it is also hard to see why issues of regularization, counterterms, sign of the coupling constant, and other issues mentioned in Section 4.2 are merely empirical. The other main contribution of Lagrangian QFT to constructive QFT is the requirement of correspondence to the renormalized perturbation series. While this has some connection to empirical success, it is also something that goes beyond mere empirical success. If we wanted to capture only the empirical content of Lagrangian QFT, all we would ask for is equivalent scattering matrices. In addition, we saw that the requirement of correspondence is not merely an "adding" of content to constructive QFT, but is constitutive of its success. It's not that constructive QFT on its own already has theoretical content without Lagrangian QFT, but to decide even what its theoretical content is, namely its solutions, we need Lagrangian QFT for guidance. In other words, even if one insists on interpreting the contribution of Lagrangian QFT as merely "empirical", it's still the case that the empirical content is guiding what counts as acceptable theoretical content. This should be unsurprising given the role of constructive QFT as essentially mediating between empirical success and as-yet-unconfirmed axiom systems.

Fourthly, constructive QFT contributes some conceptual clarity to what is going on in Lagrangian QFT. The Wightman axioms, for example, have a clear conceptual content. They decscribe a Hilbert space, and they incorporate straightforward physical principles like microcausality. This kind of physical content may be somewhat obscured in the complicated techniques of Lagrangian QFT. Constructive QFT thus serves as a bridge between the physical content of the Wightman axioms and the empirically successful techniques of Lagrangian QFT. ${ }^{23}$ Admittedly, this picture is complicated by the fact that this bridge proceeds via a detour through the OS axioms, an issue that may deserve further philosophical attention.

Finally, given the crucial role of Lagrangian QFT in setting the success conditions for a solution in constructive QFT and in guiding us as to the details of the construction, it is possible that Lagrangian QFT adds physical content to QFT,

\footnotetext{
${ }^{23}$ I thank an anonymous referee for suggesting this point.
} 
Copyright Philosophy of Science 2016

Preprint (not copyedited or formatted)

Please use DOI when citing or quoting

over and above what the various axiomatic systems say. This might serve as guidance to future, better axiomatic systems. One particular source of physical content might be the contributions of the RG. The scaling properties of fields that the $\mathrm{RG}$ reveals are not something that can be obviously read off the axioms. There is also an open question of whether the diagrammatic methods used to manage expansions in constructive QFT have any physical content. ${ }^{24}$ This is related to ongoing debates about whether Feynman diagrams in Lagangian QFT are merely a convenient calculational tool or have real physical content (Wüthrich, 2012). The lack of mathematical rigor of Lagrangian QFT may be a reason to dismiss the foundational importance of techniques associated with Lagrangian QFT, such as the $\mathrm{RG}$ and Feynman diagrams, but their continued importance even in rigorous enterprises like constructive QFT removes this reason.

\section{Conclusion}

In this paper, I highlighted two aspects of constructive QFT that have been neglected by philosophers. The first is that a solution in constructive QFT must have some kind of correspondence relation to the renormalized perturbation series of Lagrangian QFT, even though the latter is not mathematically well-defined. The second is that a successful construction depends on various choices of regularizations, counterterms, and so on, and these choices depend at least partly on information from Lagrangian QFT.

One perspective from which to view this situation is that the empirically successful formalism of Lagrangian QFT and its distance from axiomatic QFT creates a problem that constructive field theory tries to solve. The problem is to show that the "solutions" of Lagrangian QFT, which are not well-defined, can be reproduced in some sense by something that is consistent with the axioms. However, the fact that we are trying to use mathematics to reproduce something that is not mathematically well-defined suggests that the problem is not one of mathematics alone. Rather, it also concerns how we can best translate a semi-formal language, i.e. Lagrangian QFT, into rigorous mathematics. A good translation ought to reproduce the important effects of the original language, and in this case, constructive field theorists have judged that the renormalized perturbation series are worth recovering. But this is a judgment that considers extra-mathematical values concerning what the "important effects" that the translation must reproduce are. There is an analogous situation in the case of

${ }^{24}$ This question has not, to my knowledge, being tackled by philosophers yet. Diagrammatic methods are used in Rivasseau (1984) and Mack and Pordt (1985), for example. 
Copyright Philosophy of Science 2016

Preprint (not copyedited or formatted)

Please use DOI when citing or quoting

partial differential equations (PDEs) in applied mathematics. Often, there might not be a strong solution to a given PDE-a solution that is a function that is sufficiently smooth to have all the derivatives that appear in the PDE. In such cases, we often look for generalized solutions, where we expand our search for solutions to include more discontinuous options. There are many ways to expand this search. Including distributions-entities of the same kind as delta functions - is only one option. Crucially, the PDE by itself does not tell us what kind of generalized solution is appropriate. Instead, the physical situation suggests how we should define a generalized solution ${ }^{25}$ The formalism of the PDE can be conceived as applying to different types of solution spaces depending on the physical problem of interest. There is an extra-mathematical judgment of which solution space would best capture the effects of interest in that physical situation.

The point I am making has been noted elsewhere in the philosophy of mathematics. Philip Davis (2009) has argued that in mathematics generally, it is not clear that there is a homogeneous conception of what it means to solve a problem. One could also view the issue as one in which the initial problem setting, as a mere formalism, contains multiple possible mathematical interpretations. Often, finding the correct solution involves first figuring out what the correct mathematical translation of the problem setting is. Thus, in PDEs one must first figure out which function space the derivative operators are acting on, and in QFT one must figure out what the functional integral means and how it should relate to perturbative series. Vincent Rivasseau, one of the leading figures in constructive QFT, endorses this view of the flexibility of mathematical interpretations:

in mathematics non-existence theorems, although quite common, rarely remain the last word on a subject. Often a problem with no solution is simply badly formulated and has to wait until the proper formalism in which it does have a solution is found (Rivasseau, 1991, p. 271).

Constructive QFT, as the best attempt to obtain solutions modelling particular interactions in QFT, tells us the possible behavior of QFT systems with those interactions. It thus ought to be interesting to philosophers who care about what QFT says the world is like. Yet the mathematical structure of solutions in constructive QFT is not simply given by the axioms they satisfy. These solutions also have to satisfy some kind of correspondence to perturbative solutions and they

${ }^{25}$ Thus Terry Tao (], p. 1) writes of one possible definition of generalized solutions that in some situations it fails to exclude "physically implausible" solutions, so we should turn to another definition in those cases. In another example, Tao writes that in cases where we expect there to be no actual physical singularities, then we should deal with mathematical singularities in the PDE by using a regularizing parameter to define generalized solutions (Tao, , p. 2). 
Copyright Philosophy of Science 2016

Preprint (not copyedited or formatted)

Please use DOI when citing or quoting

can only be defined as limits of very specific kinds of approximations which appear to be indispensable. Thus Lagrangian QFT might add constraints to the kinds of QFT systems of interest to philosophers, constraints not to be found in the axioms. Constructive QFT also offers an interesting example of the interaction between unrigorous and rigorous mathematics, complicating the debate about whether philosophers should take Lagrangian QFT seriously. For all these reasons, constructive QFT deserves more philosophical attention.

\section{References}

Aizenman, M. (1981). Proof of the triviality of $\phi_{d}^{4}$ field theory and some mean-field features of Ising models for $d>4$. Physical Review Letters 47(1), 1-4.

Araki, H. (1972). Axiomatic field theory. In J. D. Jackson and A. Roberts (Eds.), Proceedings, 16th International Conference on High-Energy Physics, ICHEP, Batavia, Illinois, 6-13 Sep 1972, pp. 1-6.

Baker, D. J. and H. Halvorson (2010). Antimatter. The British Journal for the Philosophy of Science 61(1), 93-121.

Batterman, R. W. (2010, March). On the explanatory role of mathematics in empirical science. The British Journal for the Philosophy of Science 61(1), $1-25$.

Brunetti, R., M. Duetsch, and K. Fredenhagen (2009, July). Perturbative algebraic quantum field theory and the renormalization groups. Online preprint.

Caliceti, E., V. Grecchi, and M. Maioli (1986). The distributional Borel summability and the large coupling $\phi^{4}$ lattice fields. Communications in Mathematical Physics 104(1), 163-174.

Callaway, D. J. E. (1988, September). Triviality pursuit: Can elementary scalar particles exist? Phys. Rep. 167(5), 241-320.

Clifton, R. and H. Halvorson (2001). Are Rindler quanta real? Inequivalent particle concepts in quantum field theory. The British Journal for the Philosophy of Science 52(3), 417-470.

Davis, P. J. (2009). When Is a Problem Solved?, pp. 81-94. Washington DC: The Mathematical Association of America.

Detlefsen, M. (2005). Formalism. In S. Shapiro (Ed.), The Oxford handbook of philosophy of math and logic, pp. 236-317. Oxford University Press.

Douglas, M. R. (2011). Foundations of quantum field theory. Presentation slides at String-Math 2011.

Erdelyi, A. (2010). Asymptotic Expansions. New York: Dover Publications.

Fraser, D. (2008). The fate of 'particles' in quantum field theories with 
Copyright Philosophy of Science 2016

Preprint (not copyedited or formatted)

Please use DOI when citing or quoting

interactions. Studies In History and Philosophy of Science Part B: Studies In History and Philosophy of Modern Physics 39(4), 841-859.

Fraser, D. (2011). How to take particle physics seriously: A further defence of axiomatic quantum field theory. Studies In History and Philosophy of Science Part B: Studies In History and Philosophy of Modern Physics 42(2), $126-135$.

Fredenhagen, K., K.-H. Rehren, and E. Seiler (2006). Quantum field theory: Where we are. In I.-O. Stamatescu and E. Seiler (Eds.), Approaches to Fundamental Physics, Volume 721 of Lecture Notes in Physics, Chapter 4, pp. 61-87. Berlin: Springer.

Gallavotti, G. (1985). Renormalization theory and ultraviolet stability for scalar fields via renormalization group methods. Reviews of Modern Physics 57(2), 471-562.

Gallavotti, G. and V. Rivasseau (1984). $\phi^{4}$ field theory in dimension 4: A modern introduction to its unsolved problems. In Annales de l'IHP Physique théorique, Volume 40, pp. 185-220. Elsevier.

Gawedzki, K. and A. Kupiainen (1985). Massless lattice $\phi_{4}^{4}$ theory: Rigorous control of a renormalizable asymptotically free model. Communications in Mathematical Physics 99.

Glimm, J. (1969). The foundations of quantum field theory. Advances in Mathematics 3(1), 101-125.

Glimm, J. and A. Jaffe (1968, Dec). A $\lambda \phi^{4}$ quantum field theory without cutoffs. I. Phys. Rev. 176, 1945-1951.

Glimm, J., A. Jaffe, and T. Spencer (1974). The Wightman axioms and particle structure in the $\mathrm{P}(\phi)_{2}$ quantum field model. Annals of Mathematics 100(3), 585-632.

Halvorson, H. and R. Clifton (2002). No place for particles in relativistic quantum theories? Philosophy of Science 69(1).

Halvorson, H. and M. Müger (2006). Algebraic quantum field theory. In J. Butterfield and J. Earman (Eds.), Philosophy of Physics (Handbook of the Philosophy of Science), pp. 731-922. Amsterdam: North-Holland Publishing Co.

Jaffe, A. (1969, October). Whither axiomatic field theory? Reviews of Modern Physics 41(4), 576-580.

Jaffe, A. and E. Witten (2005). Quantum Yang-Mills theory.

http://millenniumproblems.org/Assets/pdf/ QuantumYangMillsWebRevised.pdf (accessed June 7, 2016).

Kleinert, H. and V. Schulte-Frohlinde (2001). Critical Properties of $\phi^{4}$ Theories. Singapore: World Scientific. 
Copyright Philosophy of Science 2016

Preprint (not copyedited or formatted)

Please use DOI when citing or quoting

Kline, M. (1983). Euler and infinite series. Mathematics Magazine 56(5), 307-314.

Mack, G. and A. Pordt (1985). Convergent perturbation expansions for Euclidean quantum field theory. In A. Jaffe, H. Lehmann, and G. Mack (Eds.),

Quantum Field Theory, pp. 267-298. Berlin: Springer.

Magnen, J. and R. Sénéor (1977). Phase space cell expansion and Borel summability for the Euclidean $\phi_{3}^{4}$ theory. Communications in Mathematical Physics 56(3), 237-276.

Pincock, C. (2004). A new perspective on the problem of applying mathematics. Philosophia Mathematica 12(2), 135-161.

Ramond, P. (1981). Field Theory: A Modern Primer (2nd ed.). Reading, Massachusetts: The Benjamin/Cummings Publishing Company, Inc.

Rivasseau, V. (1984). Construction and Borel summability of planar 4-dimensional Euclidean field theory. 95(4), 445-486.

Rivasseau, V. (1991). From Perturbative to Constructive Renormalization. Princeton University Press.

Rivasseau, V. (1999, February). Constructive renormalization theory. Online lecture notes. http://arxiv.org/abs/math-ph/9902023 (accessed June 7, 2016).

Rosen, L. (1973). The $\mathrm{P}(\phi)_{2}$ model. In G. Iverson, A. Perlmutter, and S. Mintz (Eds.), Fundamental interactions in physics and astrophysics, pp. 86-107. New York: Plenum Press.

Ruetsche, L. (2011). Interpreting Quantum Theories. Oxford University Press.

Salmhofer, M. (2007, October). Renormalization: An Introduction. Berlin: Springer.

Sénéor, R. (1988). Some remarks for the construction of Yang-Mills field theories. In P. Breitenlohner, D. Maison, and K. Sibold (Eds.), Renormalization of Quantum Field Theories with Non-linear Field Transformations, Volume 303 of Lecture Notes in Physics, pp. 22-31. Berlin: Springer.

Simon, B. (1982, January). Large orders and summability of eigenvalue perturbation theory: A mathematical overview. Int. J. Quantum Chem. 21(1), $3-25$.

Steinmann, O. (2000, September). Perturbative Quantum Electrodynamics and Axiomatic Field Theory. Berlin: Springer.

Strocchi, F. (2013). An Introduction to the Non-Perturbative Foundations of Quantum Field Theory. Oxford University Press.

Summers, S. J. (2012). A perspective on constructive quantum field theory. Online preprint.

Tao, T. Generalized solutions. Online preprint. http://www.math.ucla.edu/ tao/preprints/generalized_solutions.pdf (accessed June 7, 2016). 
Copyright Philosophy of Science 2016

Preprint (not copyedited or formatted)

Please use DOI when citing or quoting

Teller, P. (1997). An Interpretive Introduction to Quantum Field Theory. Princeton University Press.

Wallace, D. (2006). In defence of naiveté: The conceptual status of Lagrangian quantum field theory. Synthese 151(1), 33-80.

Wallace, D. (2011). Taking particle physics seriously: A critique of the algebraic approach to quantum field theory. Studies In History and Philosophy of Science Part B: Studies In History and Philosophy of Modern Physics 42(2), 116-125.

Wightman, A. S. (1969). What is the point of so-called "axiomatic field theory"? Physics Today 22(9), 53-58.

Wightman, A. S. (1973). Constructive field theory: Introduction to the problems. In G. Iverson, A. Perlmutter, and S. Mintz (Eds.), Fundamental Interactions in Physics and Astrophysics, Volume 3 of Studies in the Natural Sciences, pp. 1-85. New York: Springer.

Wightman, A. S. (1976). Hilbert's sixth problem: mathematical treatment of the axioms of physics. In F. A. Browder (Ed.), Mathematical Developments Arising from Hilbert Problems, pp. 147-240. Providence, Rhode Island: American Mathematical Society.

Wightman, A. S. (1986). Some lessons of renormalization theory. In J. de Boer, E. Dal, and O. Ulfbeck (Eds.), The Lesson of Quantum Theory, Amsterdam, pp. 201-226. North-Holland.

Wilson, M. (2008). Wandering Significance: An Essay on Conceptual Behaviour. Oxford University Press.

Wüthrich, A. (2012). Interpreting Feynman diagrams as visual models.

Spontaneous Generations: A Journal for the History and Philosophy of Science 6(1). 